or inadequate intake. Children with feeding problems also tended to eat less than children without feeding problems. There was a trend towards more feeding problems in patients with chromosomal abnormalities or other associated anomalies.

Conclusion Feeding disorder is often and frequent long-term sequel in children after neonatal or early infancy heart surgery. Patients with chromosomal and associated anomalies and those who underwent multiple cardiac surgeries are at risk of developing feeding difficulties and later feeding disorders. These patients need to be selected for preventive strategies and nutritional intervention should be offered in order to increase the caloric intake of the child and to develop a sound feeding relationship in the family.

\section{GP37 ECHOCARDIOGRAPHIC FOLLOW-UP OF CHILDREN WITH SUPRAVALVULAR AORTIC STENOSIS}

${ }^{1}$ Betül Çınar*, ${ }^{2}$ Sezen Ugan Atik, ${ }^{2}$ Ayşe Güler Eroğlu, ${ }^{2}$ Levent Saltık. ${ }^{1}$ Istanbul University Cerrahpaşa Medical Faculty, Department of Pediatrics, istanbul, Turkey; ${ }^{2}$ Istanbul University Cerrahpaşa Medical Faculty, Department of Pediatric Cardiology, istanbul, Turkey

\subsection{6/archdischild-2019-epa.103}

Introduction Supravalvular aortic stenosis (SVAS) is the congenital stenosis of the ascending aorta, which may be discrete or diffuse. It can be seen in children with no other underlying disease, especially in syndromic cases such as Williams syndrome. Supravalvular aortic stenosis is also seen as an autosomal dominant type of familial and sporadic cases. In this study, long-term and postoperative follow-up results of patients who were followed up between 1990 and 2017 were presented.

Materials and methods The study included 31 patients who were evaluated and followed with echocardiography at our institution between 1990 and 2017 with SVAS.

Results Twelve of the cases were female (39\%). Twenty patients were diagnosed with Williams syndrome. Thirty patients remained after exclusion of one case who was lost during cardiac catheterization, were followed for an average of $6.7 \pm 5.8$ years. The severity of SVAS detected in the first echocardiographic examination was very mild in 6 cases (19\%), mild in 8 cases (26\%), moderate in 5 cases $(16 \%)$ and severe in 12 cases (39\%). Six patients with severe SVAS were operated after the first echocardiographic examination. Right ventricular outflow tract stenosis was detected in the first examination in 6 cases and appeared in the third year of follow-up in one case. The mean right ventricular outflow tract obstruction gradient was $46.4 \pm$ $18.5 \mathrm{mmHg}$ on Doppler examination. According to the recent echocardiographic examination, the distribution of the degree of SVAS; Six patients $(19.3 \%)$ were very mild, 5 (16\%) were mild, 6 (19\%) were moderate, and $12(38.7 \%)$ were severe. SVAS progressed from very mild to mild in one patient, mild to moderate in 3 patients and moderate to severe in 3 patients. On the other hand, in 2 cases with severe SVAS were decreased during follow-up. Right ventricular outflow tract stenosis was lost in four cases and there was no change in three cases. Peripheral pulmonary stenosis (PPS) was present in 14 patients and PPS progressed in 2 patients, remained same in 7 cases and regressed in 5 cases. In the first examination, 21 of the cases had no aortic regurgitation; one patient had very mild, 5 had mild, and 5 had moderate aortic regurgitation gradually. Fourteen patients did not develop aortic regurgitation up to last examination.

Conclusion SVAS is a rare cause of left ventricular outflow tract obstruction requiring echocardiographic close follow-up

\section{GP38 SPECKLE TRACKING AS A MODERN ECHOCARDIOGRAPHIC TECHNIQUE IN ASSESSING CARDIAC FUNCTION IN CHILDREN}

${ }^{1,2}$ Andrada Mara Ardelean*${ }^{*},{ }^{1}$ Ruxandra Steflea, ${ }^{1,2}$ Ramona Stroescu, ${ }^{1,2}$ Raluca Isac,
${ }^{1,2}$ Cristina Olariu, ${ }^{1,2}$ Mihai Gafencu, ${ }^{1,2}$ Anca Popoiu, ${ }^{1,2}$ Gabriela Doros. ${ }^{1}$ Victor Babes
University of Medicine and Pharmacy, Timisoara, Romania; ${ }^{2}$ Illrd Peditric Clinic, Louis
Turcanu Emergency Hospital for Children, Timisoara, Romania

10.1136/archdischild-2019-epa.104

Introduction Speckle tracking is a new method of assessing cardiac function in adults, but used only at a research level in children. The aim is to implement this method in children with congenital and acquired cardiac pathology and oncologic disease as a new method of detecting cardiac function deterioration.

Methods and material We selected patients with congenital or acquired heard disease and oncologic pathology then performed echocardiography, cardiac biomarkers and speckle tracking and compared the results.

Results Currently we have 31 patients out of which $55 \%$ had a cardiac pathology, 26\% had an oncological disease and 19\% were collagenases or vasculitides. However, the study is ongoing and we hope to achieve a larger patient pool.

When considering the patients with congenital heart disease $47 \%$ had modified GLS(global longitudinal strain) and cardiac biomarkers, but a normal EF(ejection fraction). Aortic coarctation, aortic regurgitation, complex cyanogenic malformations, ventricular septal defect, complete AV block, hypertrophic cardiomyopathy and dilated cardiomyopathy were the maladies with altered GLS but with normal ejection fraction.

In the case of the complete AV block, she had a new dual chamber pacemaker installed, after which she developed symptoms of cardiac failure, GLS was under normal values, EF was $54 \%$ and had a septal dyssynchrony, however after modifying the device's parameters the GLS was normal and there was no septal dyssynchrony.

Patients with chemotherapy frequently develop cardiac toxicity. $44 \%$ of patients were diagnosed with cardiotoxicity with the help of speckle tracking and cardiac biomarkers.

One patient with Rhabdomyosarcoma with an initial normal GLS, chemotherapy was initiated, after which he accused palpitations. The GLS was at the lower normal limit, thus confirming cardiotoxicity due to Doxorubicin.

One patient with LES was monitored by speckle tracking. Initial normal GLS, was altered during a disease flare after which pericarditis developed.

We had two patients with Kawasaki disease complicated with severe coronary aneurysms which were followed-up by speckle tracking.

Conclusions Speckle tracking can be used as a fast and safe method to determine cardiac function in children, being well correlated with cardiac biomarkers, even when EF is normal. 